\title{
Fe XIII coronal line emission in cool M dwarfs ${ }^{\star}$
}

\author{
B. Fuhrmeister, J. H. M. M. Schmitt, and R. Wichmann
}

Hamburger Sternwarte, University of Hamburg, Gojenbergsweg 112, 21029 Hamburg, Germany

Received 7 October 2003 / Accepted 17 December 2003

\begin{abstract}
We report on a search for the Fe XIII forbidden coronal line at 3388.1 Å in a sample of 15 M-type dwarf stars covering the whole spectral class as well as different levels of activity. A clear detection was achieved for LHS 2076 during a major flare and for CN Leo, where the line had been discovered before. For some other stars the situation is not quite clear. For CN Leo we investigated the timing behaviour of the Fe XIII line and report a high level of variability on a timescale of hours which we ascribe to microflare heating.
\end{abstract}

Key words. stars: activity - stars: coronae - stars: late-type

\section{Introduction}

The solar corona can be studied at extremely high spatial and spectral resolution over almost the entire range of the electromagnetic spectrum. With typical coronal plasma temperatures of a few million degrees the bulk of the Sun's coronal energy losses is emitted in the X-ray range, the energy losses at shorter and longer wavelengths being considerably smaller. Therefore observations of the solar corona in the optical are quite difficult because of the faintness of the corona compared to the photosphere in this wavelength range. This problem can be overcome at least for observations above the solar limb if the photospheric light is blocked, e.g. by the Moon during an eclipse or - artificially - by a coronograph. Optical observations of the solar corona carried out in that fashion were the first to reveal the true nature of the corona as a hot plasma with temperatures much higher than the underlying chromosphere or photosphere. However, the solar corona could only be observed off the limb in the optical, and only through satellite-based imaging observations of the corona at X-ray and EUV wavelengths the corona at large could be observed and studied.

The existence of coronae similar to that of the Sun around essentially all late-type main sequence stars with outer convection zones has been established by extensive X-ray studies (e.g. Schmitt 1997). As is the case for the Sun, the bulk of the energy losses for stellar coronae also occurs in the X-ray range, and consequently this spectral band is the most natural one for the study of stellar coronae. However, coronal emission from stars can also be observed in the radio range (e.g. Berger 2002 or Güdel 2002) and in the optical (Schmitt \& Wichmann 2001). Coronal observations at those latter wavebands are

Send offprint requests to: B. Fuhrmeister, e-mail: bfuhrmeister@hs.uni-hamburg.de

* Based on observations collected at the European Southern Observatory, Paranal, Chile, 68.D-0166A. extremely difficult since the coronal emissions are much fainter, and especially in the optical, the weak coronal emission has to be detected above the usually much brighter optical photospheric emission. The most promising candidates to search for optical coronal emission are clearly late-type $\mathrm{M}$ dwarf stars, which can be as X-ray bright as or even X-ray brighter than the Sun, but whose photospheric emission is rather faint in particular at near UV wavelengths. The detection of coronal emission in the optical was recently accomplished for the active Mstar CN Leo, where Schmitt \& Wichmann (2001) were able to detect the Fe XIII forbidden coronal line at $3388.1 \AA$. This successful detection of coronal emission in one star raises the question to what extent such detections are possible for other cool stars as well or whether CN Leo is a unique and singular case.

In this paper we will discuss the problems of detecting the $3388.1 \AA$ forbidden coronal line in a small sample of late-type stars and present an analysis of the temporal variability of this line in the "proto-type" CN Leo. Our paper is structured as follows: In Sect. 2 we describe the VLT data used for our analyses and the sample of investigated stars. In Sect. 3 an overview over the spectral range under investigation is given, while in Sect. 4 we deal with the rotational velocity of the analyzed stars. In Sect. 5 the results of our search for the Fe XIII line for individual stars are presented and the timing behavior of the line for CN Leo is discussed in Sect. 6. Section 7 deals with the X-ray to Fe XIII line ratio for CN Leo and LHS 2076. In Sect. 8 we describe the results of our search for other forbidden coronal lines in CN Leo.

\section{Observations and data analysis}

The observations reported in this paper were obtained in visitor mode with ESO's Kueyen telescope at Paranal equipped with the Ultraviolet-Visual Echelle Spectrograph (UVES) from 
Table 1. Basic observations parameters of the observed stars.

\begin{tabular}{|c|c|c|c|c|c|c|}
\hline name & $\begin{array}{l}\text { other } \\
\text { name }\end{array}$ & $\begin{array}{l}\text { spectral } \\
\text { type }\end{array}$ & $\log L_{X}{ }^{1}$ & observations & Ti II lines & $\begin{array}{l}\text { highest } \\
\text { Balmer line }\end{array}$ \\
\hline LHS 1827 & GJ 229A & M 1 & $27.13^{a}$ & 2002-03-15 4 spectra $1200 \mathrm{~s}$ & absorption & - \\
\hline LHS 428 & & M 3e & $28.75^{a}$ & 2002-03-15 2 spectra $1200 \mathrm{~s}$ & absorption & $\mathrm{H}_{18}$ \\
\hline LHS 6158 & & M 3.5 & $28.76^{c}$ & 2002-03-15 2 spectra 2400 s & weak absorption & $\mathrm{H}_{18}$ \\
\hline LHS 5167 & AD Leo & M 3.5 & $28.92^{a}$ & $\begin{array}{l}2002-03-133 \text { spectra } 1800 \mathrm{~s} \\
2002-03-162 \text { spectra } 1200 \mathrm{~s}\end{array}$ & emission & $\mathrm{H}_{18}$ \\
\hline HD 196982 & AT Mic & M 4.5 & $29.55^{c}$ & 2002-03-16 2 spectra $2400 \mathrm{~s}$ & emission & $\mathrm{H}_{20}$ \\
\hline LHS 1943 & YZ CMi & $\mathrm{M} 4.5 \mathrm{e}$ & $28.67^{b}$ & 2002-03-13 3 spectra 3600 s & emission & $\mathrm{H}_{24}$ \\
\hline LHS 2664 & FN Vir & M 4.5 & $27.92^{b}$ & 2002-03-13 3 spectra $3600 \mathrm{~s}$ & weak emission & $\mathrm{H}_{18}$ \\
\hline LHS 324 & GL Vir & M 5 & $27.65^{a}$ & $\begin{array}{l}2002-03-133 \text { spectra } 3600 \mathrm{~s} \\
2002-03-162 \text { spectra } 2400 \mathrm{~s}\end{array}$ & weak emission & $\mathrm{H}_{19}$ \\
\hline LHS 36 & CN Leo & M 5.5 & $27.78^{a}$ & $\begin{array}{l}2002-03-136 \text { spectra } 7200 \mathrm{~s} \\
2002-03-144 \text { spectra } 4800 \mathrm{~s} \\
2002-03-156 \text { spectra } 7200 \mathrm{~s} \\
2002-03-166 \text { spectra } 7200 \mathrm{~s} \\
2001-01-061 \text { spectrum } 3120 \mathrm{~s}\end{array}$ & emission & $\mathrm{H}_{24}$ \\
\hline LHS 2076 & EI Cnc & M 5.5 & $27.60^{a}$ & $\begin{array}{l}2002-03-154 \text { spectra } 4800 \mathrm{~s} \\
2002-03-161 \text { spectrum } 1200 \mathrm{~s}\end{array}$ & - & $\mathrm{H}_{18}$ \\
\hline LHS 49 & Prox Cen & M 5.5 & $27.26^{a}$ & 2001-02-02 1 spectrum 3120s & weak emission & $\mathrm{H}_{18}$ \\
\hline LHS 10 & UV Cet & M 5.5 & $27.31^{a}$ & 2000-12-17 1 spectrum 3120 s & emission & $\mathrm{H}_{18}$ \\
\hline LHS 248 & DX Cnc & M 6 & $26.60^{a}$ & 2002-03-16 3 spectra 3600 s & - & $\mathrm{H}_{11}$ \\
\hline LHS 2034 & $\mathrm{AZ} \mathrm{Cnc}$ & M 6 & $28.89^{c}$ & $\begin{array}{l}2002-03-146 \text { spectra } 6000 \mathrm{~s} \\
2002-03-162 \text { spectra } 2400 \mathrm{~s}\end{array}$ & emission & $\mathrm{H}_{17}$ \\
\hline LHS 292 & & M 6.5 & & 2001-02-02 1 spectrum 3120s & - & $\mathrm{H}_{15}$ \\
\hline
\end{tabular}

${ }^{1}$ In erg $\mathrm{s}^{-1}$.

${ }^{a}$ Schmitt et al. (1995).

${ }^{b}$ Delfosse et al. (1998).

${ }^{c}$ Hünsch et al. (1999).

${ }^{d}$ Schmitt \& Liefke (2002) X-ray luminosity is variable.

March, 13th to 16th in 2002, with the exception of UV Ceti, Prox Cen, LHS 292 and one of the CN Leo spectra, which were observed in service mode during the winter season 2000/2001. For the March 2002 run the instrument was operated in a dichroic mode, yielding 33 echelle orders in the blue arm (spectral coverage from 3030 to $3880 \AA$ ) and 39 orders in the red arm (spectral coverage from 4580 to $6680 \AA$ ). For the runs in the winter 2000/2001 a monochroic setup was used, providing us only with the blue part of the spectrum. In the dichroic setup the red part of the spectrum is recorded on two separate CCDs; therefore there is a spectral gap from $\sim 5640$ to $5740 \AA$, resulting from a spatial gap between the two CCDs. As a consequence of the various instrumental constraints we cannot observe the lines from $\mathrm{H}_{3}$ up to $\mathrm{H}_{8}$ of the Balmer series, nor do we cover the $\mathrm{Ca}$ II $\mathrm{H}$ and $\mathrm{K}$ lines. The typical resolution of our spectra is $\sim 45000$, typical exposures times were 20 min except for the brighter among our sample stars. Normally for each star three exposures were taken in sequence in order to facilitate the recognition of cosmics, again with the exception of the four spectra of CN Leo, UV Ceti, Prox Cen and LHS 292 taken during the winter season 2000/2001.
A list of the observed stars and the available spectra for each star is provided in Table 1, giving the observation dates and total exposure times. Moreover we provide some basic parameters of the sample stars, including the highest detected Balmer line in our data as an estimator of activity. Note that during the March 2002 run CN Leo was observed nightly to carry out an investigation of its chromospheric and coronal variability.

All data were reduced using IRAF, including flat-fielding, order definition and scattered light subtraction. The wavelength calibration was carried out using Thorium-Argon spectra with an accuracy of $\sim 0.03 \AA$ in the blue arm and $\sim 0.05 \AA$ in the red arm. In addition there are photometric data from the UVES exposure meter taken for engineering purposes and therefore not flux calibrated. Still, these data were useful to assess whether the star was observed during quiescence or during a major flare.

The spectral line fits were carried out with the CORA fit program (Ness \& Wichmann 2002). This software tool was originally developed for analyzing high resolution X-ray spectra, but the fit algorithms employed by CORA are also well suited for the modeling of well-defined chromospheric and coronal emission lines. The program provides an accurate error 
analysis. All fits were carried out using Gaussian line profiles after shifting the wavelength to the stars' rest frame.

\section{Detection of the Fe XIII line}

The prime target of our observing program was the spectral region around $3388 \AA$. The specific transition under consideration is $3 s^{2} 3 p^{2}{ }^{3} P_{2}-{ }^{1} D_{2}$. Flower \& Pineau des Forets (1973) give a detailed discussion of the atomic physics of Fe XIII and provide level population calculations for a range of temperatures and densities, considering direct excitation due to collisions with electrons and protons and indirect excitation of allowed transitions via collisions with electrons followed by radiative decay and find rather low dependence on temperature.

In order to give an overview of the nature and quality of our spectra, we show (in Fig. 1) the spectral range between $3370 \AA-3390 \AA$ for most of our program stars. LHS 292 is not shown since its spectrum is very similar to DX Cnc, and LHS 428 and LHS 6158 are not shown since they are both double stars with very complicated spectra that could not be disentangled and were therefore excluded from the analysis. The spectra shown in Fig. 1 are sorted by decreasing spectral type. One notes a distinct sequence from "early" $M$ dwarfs to "late" M dwarfs. In the "early" M dwarfs like Gl 229A and $\mathrm{AD}$ Leo one still recognizes a clear photospheric spectrum in the range $3370 \AA-3390 \AA$ with some additional chromospheric emission lines, while in the later type M dwarfs like CN Leo and the (flaring) LHS 2034 an almost pure emission line spectrum appears; these latter spectra are obviously dominated by the stellar chromospheres rather than their photospheres.

The Fe XIII line at $3388.1 \AA$ detected in CN Leo by Schmitt \& Wichmann (2001) is - unfortunately - blended with a Ti II chromospheric line at $3387.846 \AA$. This particular Ti II line and other emission lines from the same multiplet are present in all recorded spectra besides the non-flare spectra of LHS 2034 and LHS 2076 and the spectrum of DX Cnc. In GL 229A Ti II appears to be present in absorption.

The stars earlier than M 4.5 have a rather high pseudocontinuum in the considered wavelength range which is clearly dominated by overlapping absorption lines. This continuum strongly decreases for the spectral types M 3.5-M 4.5 stars by a factor of ten, accompanied by the vanishing of the strong absorption features in this regime. Consequently, a detection of the Fe XIII line should be much easier for spectral types later than M 4.5. Therefore the most promising search for coronal line emission can be made for stars in the spectral range M 4.5-M 6 since for later stars even the normally strong chromospheric Ti II emission lines cannot be detected any more.

\section{Determination of rotational velocities}

A critical issue for the successful separation of the chromospheric Ti II emission line at $3387.846 \AA$ and the coronal Fe XIII emission line at 3388.1 $\AA$ are the intrinsic line broadening profiles of these lines. The wavelength difference between those lines $(\approx 0.26 \AA)$ corresponds to a velocity of $\approx 23 \mathrm{~km} \mathrm{~s}^{-1}$, easily within the reach of thermal and rotational velocities. Obviously, the thermal broadening profiles differ substantially because of the different origins of these lines, and rapid rotation is expected at least for the more active stars. Since we clearly need accurate values for $v \sin i$ for disentangling the Ti II and $\mathrm{Fe}$ XIII emission lines, we decided to measure it for some of our program stars. In order to determine $v \sin i$ we used 18 of the red arm orders that show no strong emission lines either from the star or from terrestrial airglow. As a template we decided to use CN Leo since CN Leo is known to rotate very slowly ( $v \sin i<2.9 \mathrm{~km} \mathrm{~s}^{-1}$, Delfosse et al. 1998). The measured spectrum of CN Leo was spun up with rotational velocities from 3 up to $45 \mathrm{~km} \mathrm{~s}^{-1}$. The best fit value for the rotation velocity $v \sin i$ of the spun up template and the star under study was determined with a $\chi^{2}$-test for every order used. The final rotational velocity was determined by averaging the values obtained in the different orders. We mostly used only the red part of the spectrum since in the blue part of the spectrum it is rather difficult to find larger regions unaffected by emission lines. We tried to avoid this problem of the blue spectra by using only very narrow spectral ranges $(\sim 10 \AA)$ of every aperture, yet the issues of which lines are in emission, which show emission cores and which are purely in absorption are very different for each program star. Therefore the template has to be very similar to the test star and the used wavelength intervals have to be determined individually but even then the scatter for the wavelength intervals is bigger than in the red arm. Therefore we determined the rotational velocity for only three stars in the blue arm. For these stars we used Prox Cen as template since its spectrum is very similar to these stars. CN Leo could not be used as template because it is much more active than the measured stars. The rotational velocities determined in this fashion are listed in Table 2. In general, the values determined by us agree with previously determined ones found in the literature with the exception of GL Vir, where we find a substantially larger rotational velocity both in the red and blue arm than Delfosse et al. (1998). A careful visual inspection and comparison of the spectra of GL Vir with those obtained for FN Vir and AD Leo shows a very high similarity to the faster rotating spectrum. Moreover we used a template star very similar to GL Vir in spectral type while Delfosse et al. used a synthetic K0III spectrum. Therefore we do favor our (larger) rotational velocity.

For the double star UV Cet we measure a rotational velocity of $25.9 \pm 8.6 \mathrm{~km} \mathrm{~s}^{-1}$ which agrees with the value found by Mohanty \& Basri (2003) for UV Cet B. Since UV Cet A has a similar rotation velocity (G. Basri, private communication) it cannot be decided from the rotational velocity which component of UV Cet dominates our spectrum.

\section{Observations of individual stars}

Since the spectral properties of our sample stars are very different we applied specific methods for the individual stars for a detection or non-detection of the Fe XIII line. These are discussed in detail below. 


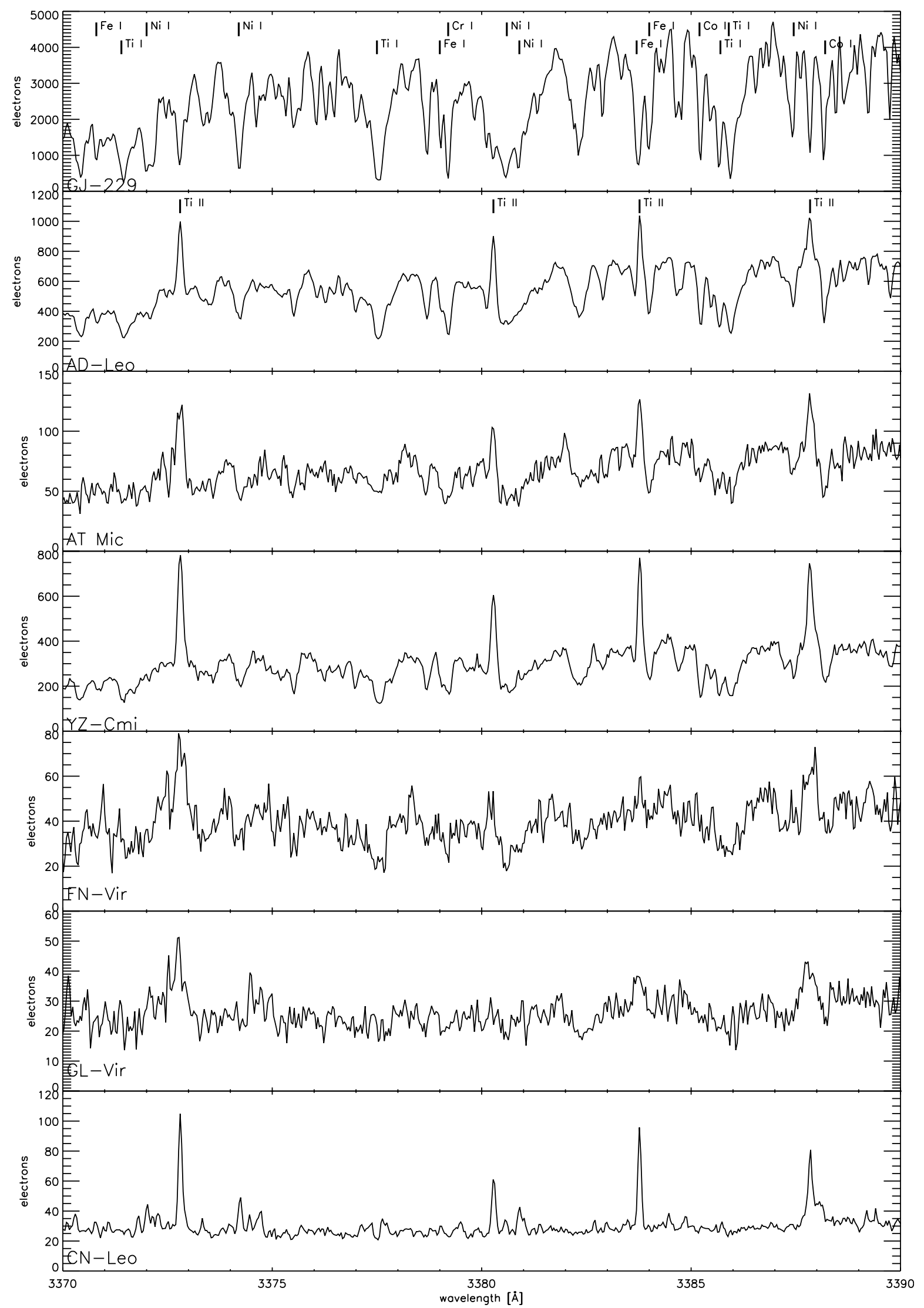

Fig. 1. The considered wavelength range of selected program stars. Some strong absorption lines are identified in the top spectrum. The Ti II emission lines are marked in the spectrum of Ad Leo. 


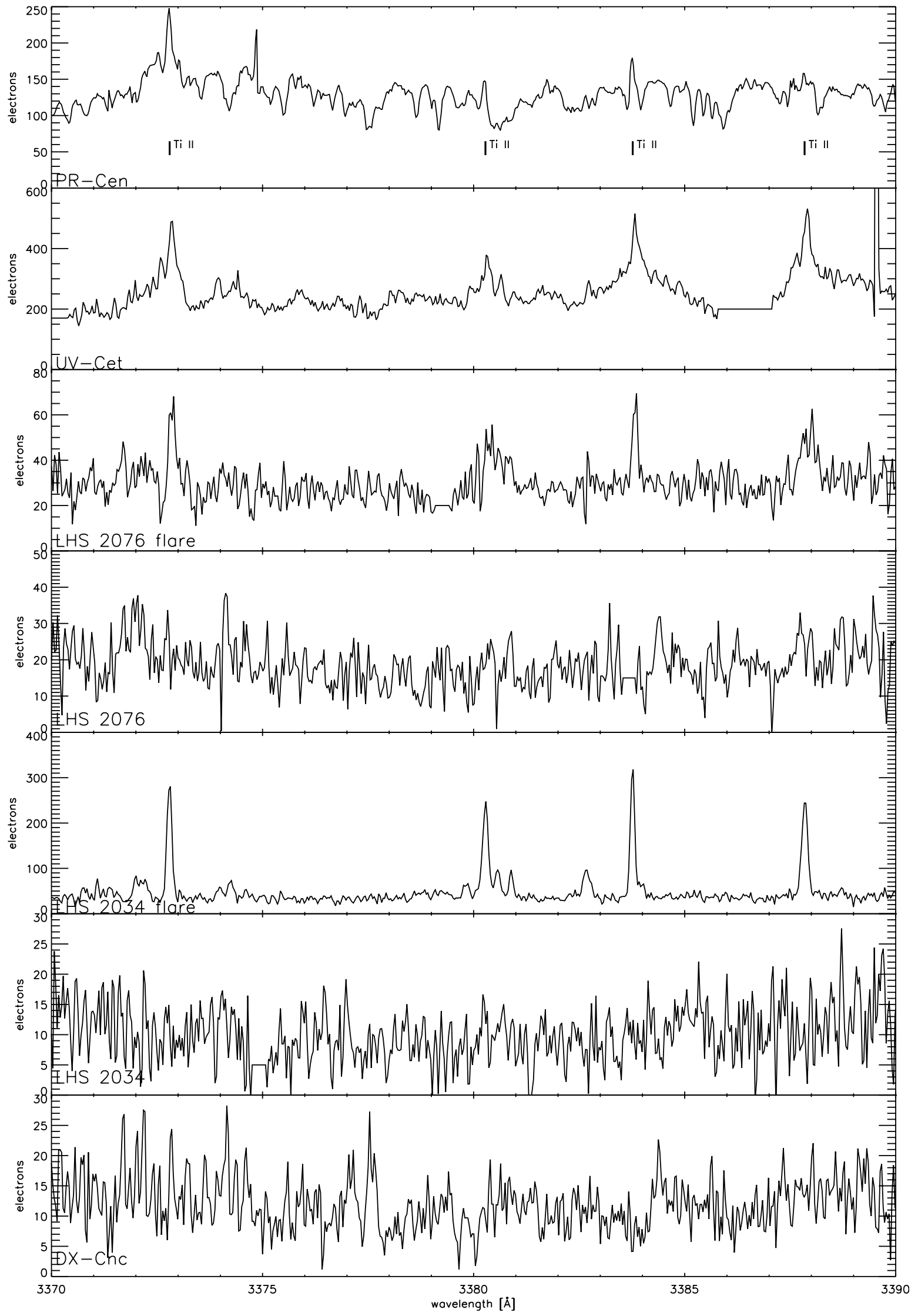

Fig. 1. continued. For LHS 2034 and LHS 2076 the spectra are shown during flare and during quiescent state. Note that these four spectra and the spectrum of UV Cet are not averaged spectra and therefore some remaining cosmics were replaced manually by a straight line. 

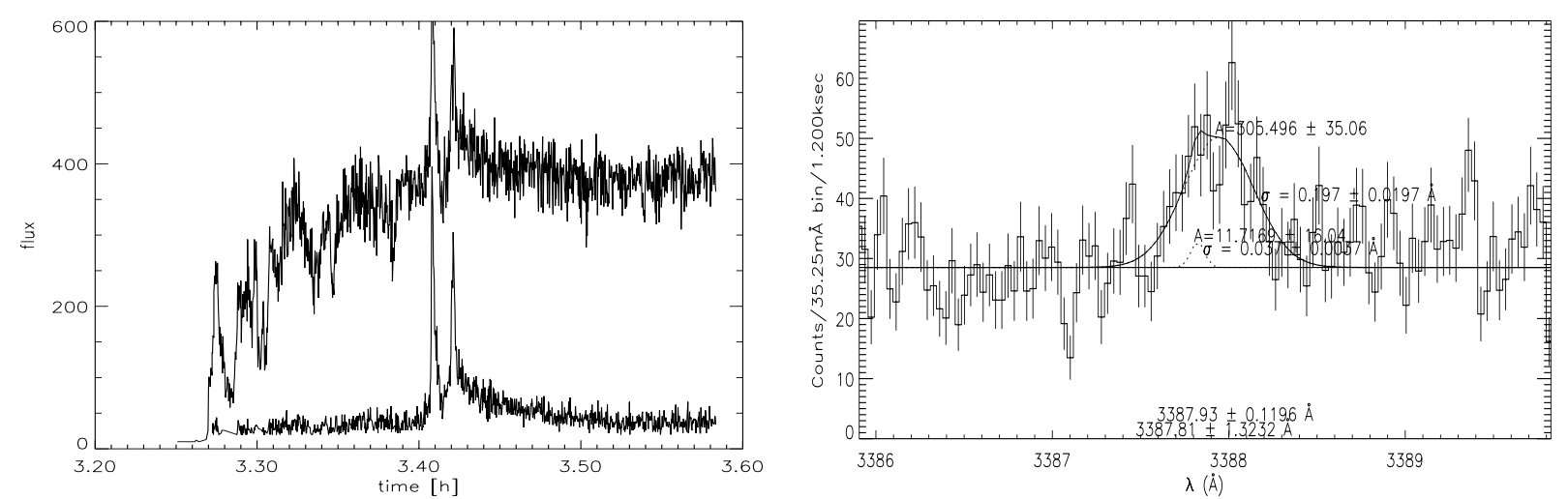

Fig. 2. To the left the light curve of the flux of LHS 2076 on March, 15th, corresponding to a single spectrum. On the $x$-axis the universal time is given. The flux is in arbitrary units since the photometer is used for engineering purposes. The upper light curve corresponds to the red arm of the spectra, while the lower light curve corresponds to the blue arm and is scaled relative to the red flux for convenience. Two short duration flares can be seen in both the red and the blue arm of the photometer following each other rapidly. To the right the spectrum of LHS 2076 during the flare around $3388 \AA$ showing an broad emission feature at $3387.92 \AA$.

Table 2. Rotational velocities for (some of) our program stars. LHS 428 is omitted because it is a double star and could not be separated. LHS 6158 even seems to be a triple system. GJ 229A is saturated in the red arm. Prox Cen was used as template for UV Cet, FN Vir and GL Vir in the blue arm. All measurements in $\mathrm{km} \mathrm{s}^{-1}$.

\begin{tabular}{cccc}
\hline \hline star & $\begin{array}{c}v \sin i \\
\text { red arm }\end{array}$ & $\begin{array}{c}v \sin i \\
\text { blue arm }\end{array}$ & literature \\
\hline AD Leo & $7.6 \pm 1.2$ & & $6.2 \pm 0.8^{1}$ \\
AT Mic & $11.7 \pm 0.9$ & & \\
YZ CMi & $6.7 \pm 0.6$ & & $7.0 \pm 2.0^{2}, 6.5 \pm 1.1^{1}$ \\
FN Vir & $17.4 \pm 1.4$ & $13.1 \pm 3.7$ & $16.8 \pm 2.1^{1}$ \\
GL Vir & $15.3 \pm 0.8$ & $16.0 \pm 2.0$ & $9.2 \pm 1.9^{1}$ \\
UV Cet B & & & $32.5 \pm 2.0^{2}$ \\
UV Cet A & - & $25.9 \pm 8.6$ & \\
DX Cnc & $8.9 \pm 0.8$ & & $11.0 \pm 2.0^{2}, 8.1 \pm 1.1^{1}$ \\
LHS 2034 & $7.9 \pm 2.8$ & & \\
\hline
\end{tabular}

${ }^{1}$ Delfosse et al. (1998).

${ }^{2}$ Mohanty \& Basri (2003).

\subsection{CN Leo}

We report a clear detection of the Fe XIII line for CN Leo, which is also found to be variable on a timescale of hours, as will be discussed in Sect. 6. Thus the Fe XIII line detection of Schmitt \& Wichmann (2001) is fully confirmed.

\subsection{LHS 2076}

A clear detection of the Fe XIII $3388.1 \AA$ emission line has been obtained during a flare on LHS 2076. LHS 2076 is a double star known for its flare activity and separated by 3.'4 with both components being late-type (Pettersen 1985). Our spectrum refers to one system component with some minor contribution from the second star. During the last of the four exposures on March, 15th a double-peaked flare can be recognized in the photometer light curve as shown in Fig. 2.
Outside the flare interval no significant Ti II emission is detectable in the spectra. The emission line properties of the flare spectrum are listed in Table 3. The emission feature at $3387 \AA$ is quite broad and was fitted with a double line component with amplitude, central wavelength and FWHM for both components as free parameters. The other Ti II lines were fitted as single lines with all three parameters $\left(\lambda_{\text {cen }}, A\right.$ and $\left.\sigma\right)$ free. Inspecting the properties of these three Ti II lines (cf., Table 3) one finds that the fitted half widths of all three lines agree very well with each other as expected for lines from the same multiplet. The half width of the narrow component of the $3387 \AA$ line also agrees well with the half widths of the three Ti II lines while the half width of the broad component is more than three times larger. We therefore conclude that the narrow component of the broad emission feature at $3387 \AA$ must be the fourth Ti II line in the multiplet, while the broad component has to be identified with the Fe XIII line. Further evidence for this interpretation can be found from the lines' amplitudes. Atomic data available through the NIST atomic spectra database ${ }^{1}$ for these lines predict equal relative intensities for the two lines at 3372.80 and $3383.77 \AA$ and equal relative intensities for the two lines at 3380.28 and $3387.84 \AA$ lowered by a factor of four compared to the former two lines. According to Table 3 the amplitudes of the 3372.80 and $3383.77 \AA$ line agree to within the errors and the two other lines are weaker. Therefore the broad component cannot be attributed to Ti II since its amplitude is more than twice as large as that of the 3372.80 or $3383.77 \AA$ lines. On the other hand, both amplitudes and half widths fit perfectly if the broad emission component is identified with the Fe XIII line; also, the fitted half widths agree very well with the half width determined for the Fe XIII line in the CN Leo time series (see Sect. 6). The central wavelength of the absolute line position is a bit on the blue side but well within the 2- $\sigma$ error, while the absolute positions of the three single fit Ti II lines seem to be slightly redshifted (but only to within $1-\sigma$ error each). The $3387.84 \AA$ Ti II line unfortunately has a large error in its central wavelength. Given this situation, we cannot draw

\footnotetext{
1 Available via http://physics.nist.gov/cgi-bin/ AtData/main_asd
} 
Table 3. Properties of the Ti II and the Fe XIII line during the flare on LHS 2076. Given are the amplitude (in electrons), the central wavelength and half width $\sigma$ of each line for the best fit. For the $3380.28 \AA$ line no error estimation for the half width was possible due to the low signal to noise ratio.

\begin{tabular}{cccccc}
\hline \hline & Ti II $(3372.80 \AA)$ & Ti II $(3380.28 \AA)$ & Ti II $(3383.77 \AA)$ & Ti II $(3387.84 \AA)$ & Fe XIII $(3388.1 \AA)$ \\
\hline amplitude & $134.3 \pm 19.2$ & $67.3 \pm 17.3$ & $115.1 \pm 17.3$ & $11.7 \pm 16.0$ & $305.5 \pm 35.1$ \\
central $\lambda$ & $3372.85 \pm 0.05$ & $3380.33 \pm 0.15$ & $3383.81 \pm 0.04$ & $3387.81 \pm 1.3$ & $3387.93 \pm 0.1$ \\
$\sigma$ & $0.06 \pm 0.01$ & 0.06 & $0.05 \pm 0.01$ & $0.04 \pm 0.01$ & $0.20 \pm 0.02$ \\
\hline
\end{tabular}

any meaningful conclusions about possible wavelength shifts and remark that during a flare line shifts frequently occur due to mass motions.

\subsection{GL Vir}

The continuum for GL Vir is low as for CN Leo, but unlike CN Leo the TiII at $3380 \AA$ line is barely detectable. Nevertheless there is a broad emission line at 3387.81 $\pm 0.14 \AA$ with a half width of $0.15 \AA$ and an amplitude of $141.7 \pm$ 28.9 counts. If this emission is attributed to the Ti II line, the measured amplitude would contradict the atomic data, which predicts the same intensity as for the $3380 \AA$ line (which is not seen at all, cf. Fig. 1). If, on the other hand, this feature is identified with the Fe XIII line, it is clearly blue shifted. Since the star is in a quiescent state (as can be seen from the light curve, which is not shown here) it is unlikely that a blue shift is caused by mass motions. However, the blue shift could alternatively be caused by absorption at the red wing of the Fe XIII line due to a Co I line at $3388.17 \AA$ which is also seen in other spectra (see Fig. 1). More evidence for an interpretation involving the Fe XIII line can be gained from the amplitude of the $3383.71 \AA$ line of $65 \pm 15$ counts. The $3387 \AA$ Ti II line should have a smaller amplitude according to the atomic data and the measured spectra of CN Leo. On the other hand, the half widths of the $3383.71 \AA$ line $(0.13 \pm 0.02 \AA)$ and of the $3387.81 \AA$ line $(0.15 \pm 0.01)$ are consistent with each other. The Ti II line at $3372.73 \AA$ is unfortunately too deformed to allow any conclusions on its half width or amplitude. Therefore the question arises whether the half widths of the two lines at $3383.71 \AA$ and $3387.81 \AA$ can be caused by rotation. The rotation velocity $v \sin i$ for GL Vir was measured by Delfosse et al. (1998) as $9.2 \pm 1.9 \mathrm{~km} \mathrm{~s}^{-1}$, while we determined $15.3 \pm 0.8 \mathrm{~km} \mathrm{~s}^{-1}$ (see Sect. 4). Thus, if our rotational velocity is correct, the half widths $\sigma$ can be explained by rotational broadening, however, if $v \sin i=9.2 \mathrm{~km} \mathrm{~s}^{-1}$, the measured half width is a somewhat too large to be explained by the rotation. So the data of GL Vir are not unambiguous.

\subsection{LHS 2034}

The flare star LHS 2034 was observed during a longer flare on March, 14th, lasting over half an hour as can be seen from the light curve of the photometer (not shown here). While only the very strongest chromospheric emission lines can be seen in quiescence, a multitude of emission lines becomes visible in the spectrum taken during flare maximum. Indeed, the flare spectrum of LHS 2034 is very similar to the spectrum

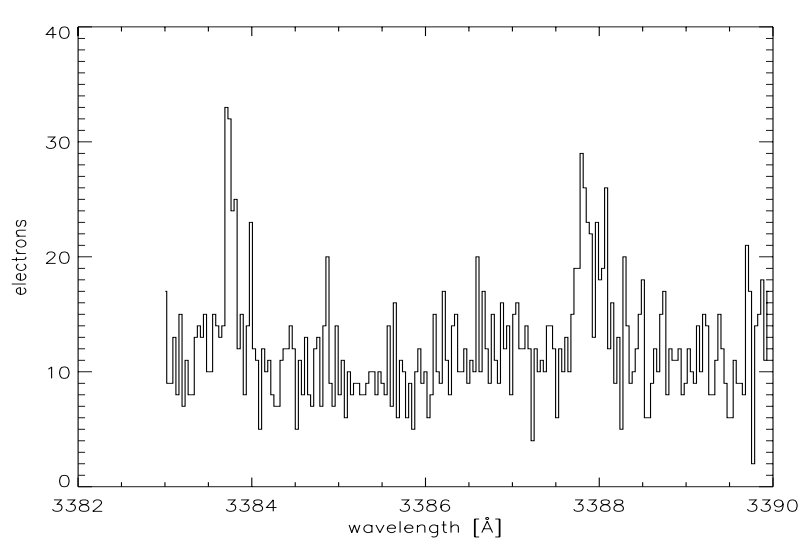

Fig. 3. Spectrum of CN Leo with a signal to noise ratio artificially reduced to that of DX Cnc. But despite the low signal to noise ratio clearly the Ti II and Fe XIII lines can be recognized.

of CN Leo with a rather flat continuum without strong absorption lines and dominated by strong chromospheric emission lines. Surprisingly, however, we did not find any evidence for Fe XIII emission in this spectrum. The same analysis procedure as applied for LHS 2076 was used for the flare spectrum of LHS 2034. All four Ti II lines are found to have about the same $F W H M$ as expected but also the same amplitude contrary to expectation from atomic data and what was found for CN Leo and LHS 2076. This fact remains unexplained. In particular, we cannot attribute - as in LHS 2076 the excess emission to Fe XIII, since this would contradict the measured $F W H M$. Obviously, efficient chromospheric heating of LHS 2034 does occur during the flare, but the corona is either not hot enough or too hot for producing a detectable Fe XIII line flux.

\subsection{Cnc and LHS 292}

These two stars and LHS 2034 are the latest-type stars in the sample and have a very low signal to noise ratio (which applies for the quiescent spectrum for LHS 2034 as well) which can cause the non detection of Fe XIII. In order to answer this question we took the spectrum of CN Leo as a template and reduced it to the lower signal to noise ratio found in our DX Cnc and the quiescent LHS 2034 spectra. The results of this exercise for DX Cnc are shown in Fig. 3. Clearly the Ti II and Fe XIII lines can be recognized in this example. Carrying out a larger number of such simulations we found that the Fe XIII need not always be recognisable, but the Ti II line should always be found in lower SNR spectra. Thus the level of activity in these stars must be lower making the persistent presence of the Fe XIII line unlikely. 
Table 4. Properties of the Ti II of UV Cet measured with CORA using a broad and a narrow component simultaneously. Given are always the values for the narrow (first) and the broad component (second).

\begin{tabular}{|c|c|c|c|c|}
\hline & Ti II (3372.80 ̊) & Ti II (3380.28 ̊) & Ti II (3383.77 ̊̊) & Ti II (3387.84 ̊) \\
\hline amplitude & 380 and 2416 & 329 and 1394 & 662 and 4665 & 520 and 5393 \\
\hline central $\lambda$ & 3372.80 & 3380.31 & 3383.82 & 3387.88 \\
\hline$\sigma$ & 0.04 and 0.23 & 0.05 and 0.30 & 0.07 and 0.53 & 0.05 and 0.46 \\
\hline
\end{tabular}

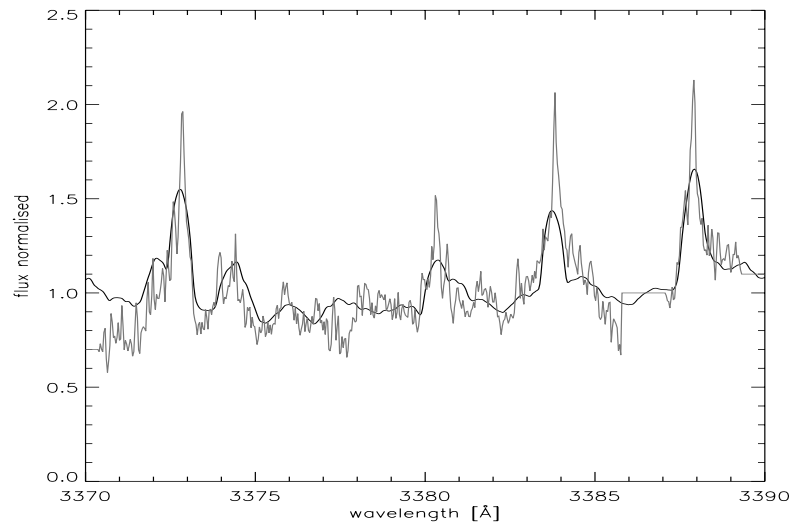

Fig. 4. The spectrum of UV Cet $3388.1 \AA$ (grey) in comparison to the spectrum of CN Leo (black) broadened artificially to the rotational velocity of UV Cet.

\subsection{UV Cet}

UV Cet is a binary flare star well known from the radio to X-ray band (Stepanov et al. 1995) and has been resolved in the X-ray for the first time only recently (Audard et al. 2003). Our UVES spectrum was taken with both components in the slit, but we were unable to resolve the spectra of the two stars (due to the seeing). Therefore the spectrum should be dominated by the brighter A-component. The emission lines seen in the spectrum of UV Cet seem to have superimposed a second component of narrow emission lines which are slightly redshifted. We tentatively interpret this second set of emission lines as an active chromospheric region occupying only a part of the stars surface, since it is too narrow to be ascribed to the second component of the binary system.

Searching for the Fe XIII line we compared the broad component of the spectrum of UV Cet with an artificially broadened spectrum of CN Leo (see Fig. 4). The Fe XIII line disappears in the broadened spectrum since it becomes totally blended with the Ti II line at $3387.84 \AA$. Therefore a detection of the Fe XIII line in the UV Cet spectrum can only be obtained through indirect reasoning as follows: first, the broadened spectrum of $\mathrm{CN}$ Leo fits very well to the UV Cet spectrum in this wavelength region, although - admittedly - in other spectral regions there is less similarity. Second, we know from CN Leo and atomic data that the flux in the Ti II line at $3387.84 \AA$ should be less than that contained in the $3372.80 \AA$ line, but for UV Cet we find just the opposite. In order to determine line fluxes, we fitted each spectral line simultaneously with a narrow and a broad component (see Table 4). If the broad component comes from the whole surface of the star and the narrow component from an active region, the combined amplitudes must be considered and the combined amplitude of the $3387.84 \AA$ line is higher than the amplitude of the $3372.80 \AA$ line thus providing
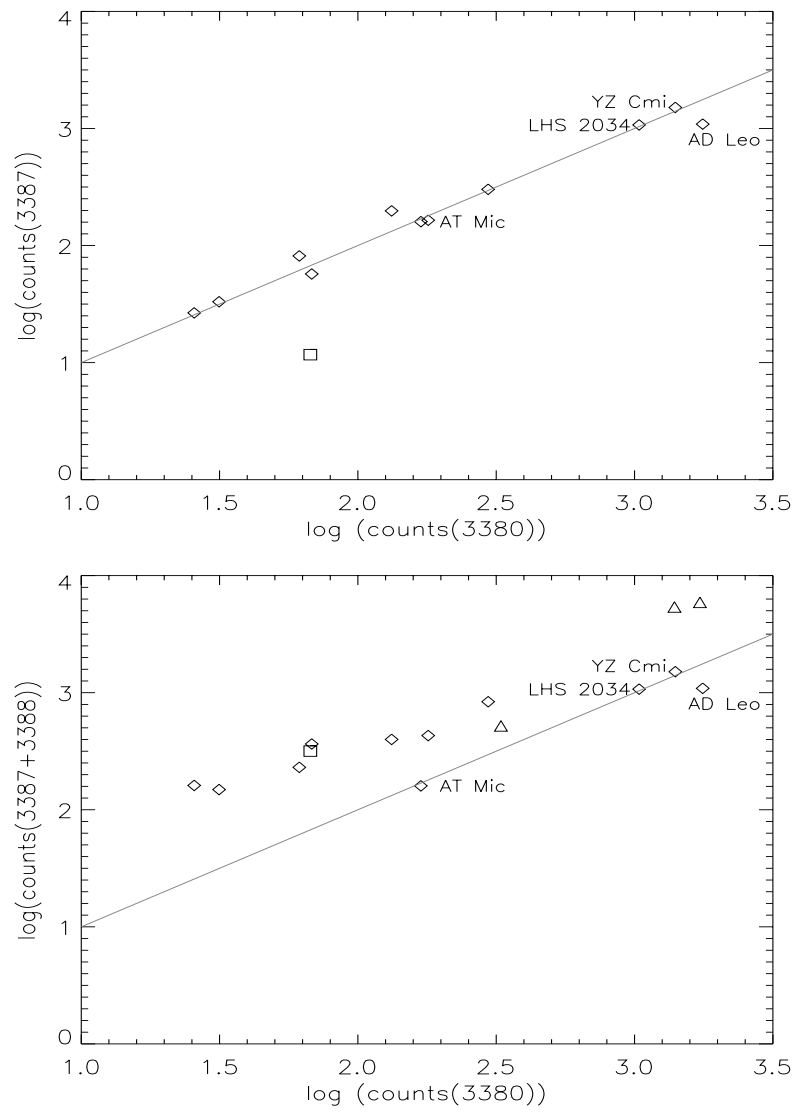

Fig. 5. Top panel: logarithm of the line flux (in electrons) of the $3387 \AA$ Aline plotted versus the logarithm of the line flux of the $3380 \AA$ line for the spectra of the CN Leo time series, LHS 2034, LHS 2076 during the flare, YZ CMi, AD Leo, AT Mic. The straight line marks the ratio of unity. The square marks the flux of LHS 2076 where the line is blended with Fe XIII and the fit cannot disentangle the two lines well. Bottom panel: logarithm of the combined flux of the $3387 \AA$ and the Fe XIII line plotted versus the $3380 \AA$ line. The triangles denote UV Cet with respect to the combined set of emission lines. Clearly AD Leo, AT Mic, YZ CMi and LHS 2034 show no Fe XIII line emission, while the UV Cet line ratios are in the regime of stars with additional Fe XIII flux (note, that the square denoting LHS 2076 has moved above the line of unity).

evidence of additional emission in the lines. The same applies for a comparison with the Ti II line at $3380.28 \AA$ for which one expects equal flux ratios. If one plots the line ratios for the time series of CN Leo and other stars, one finds, that the UV Cet lines are the only one with a ratio that needs additional flux in the $3387.84 \AA$ line (see Fig. 5). Given the high degree of activity on UV Cet it is suggestive to attribute this additional emission to the Fe XIII line, but given the complicated situation with the narrow and broad set of emission lines we consider the detection of Fe XIII in UV Cet as tentative. 


\subsection{AD Leo, AT Mic, YZ CMi, and FN Vir}

For the other stars in our sample with spectral type M 4.5-M 6 the situation is not as clear due to the presence of two absorption lines at 3387.45 (Ni I) and 3388.17 (Co I) $\AA$ in the spectrum. This situation applies for the three stars AT Mic, YZ CMi and AD Leo: the Ti II lines at 3372, 3380 and $3383 \AA$ have a width of about $0.05 \AA$ whereas the line at $3387 \AA$ shows a width of $0.1 \AA$. This can be due to the Fe XIII line blended by the absorption feature at $3388.17 \AA$, alternatively, leftover continuum may also be an explanation for the broader Ti II line since the spectra of the three stars do show many absorption lines. In order to test this hypothesis we used the spectrum of the earliest M dwarf GJ 229 as a template for the photospheric part of the spectrum applying the spun up and scaled spectrum as a "background" for the emission line fit with CORA. For the three stars AT Mic, YZ CMi and AD Leo this method yields a best fit with a narrow Ti II line of about $0.05 \AA$ half width $\sigma$, consistent with the half width found for the other Ti II lines in these stars. The fitted spectra are displayed in Fig. 6. Therefore the apparent width of the $3387 \AA$ line seems to be due to photospheric continuum and there is no need to invoke Fe XIII emission in these three stars.

For the case of FN Vir this method cannot be used, because the template GJ 229 deviates significantly from the photospheric absorption lines seen in FN Vir. However, since the Ti II line at $3372 \AA$ is found to have the same half width $\sigma$ $(0.11 \pm 0.01 \AA)$ as the $3387 \AA$ line $(0.12 \pm 0.01 \AA)$, there is again no need to invoke any Fe XIII emission for this star, either.

\subsection{Proxima Centauri}

For Proxima Centauri the Ti II lines are clearly seen in emission, but again the template GJ 229 deviates significantly if overlaid to the spectrum. In addition to this, the continuum is ill defined and therefore a fit of the line very difficult although especially the Ti II line at $3387 \AA$ is clearly seen. Although the bump on the red side of the Ti II line at $3387.84 \AA$ is somewhat suggestive of a Fe XIII emission line blended with the absorption line of Co I, this could also be due to leftover continuum. Since we were not able to obtain meaningful fits, the situation for Proxima Centauri must remain open and we must refrain from drawing any conclusions.

\section{Timing behavior of the Fe XIII line in CN Leo}

Every night during the observation run in March 2002 (with the exception of March, 14) two series of spectra were taken of CN Leo, each consisting of 3 spectra with 20 min exposure. On March, 14th only one spectral series of one hour duration was taken, one single spectrum taken about $2 \mathrm{~h}$ later was combined with the others to only one time series. We averaged the spectra of each series to improve the signal to noise ratio and constructed a total of 7 spectra of CN Leo. During the second spectral series on March, 16th a major flare occurred as can be seen in the photometer light curve in Fig. 7; on March, 14th CN Leo was very quiet, showing very little of the flickering seen in the light curves on the other days.
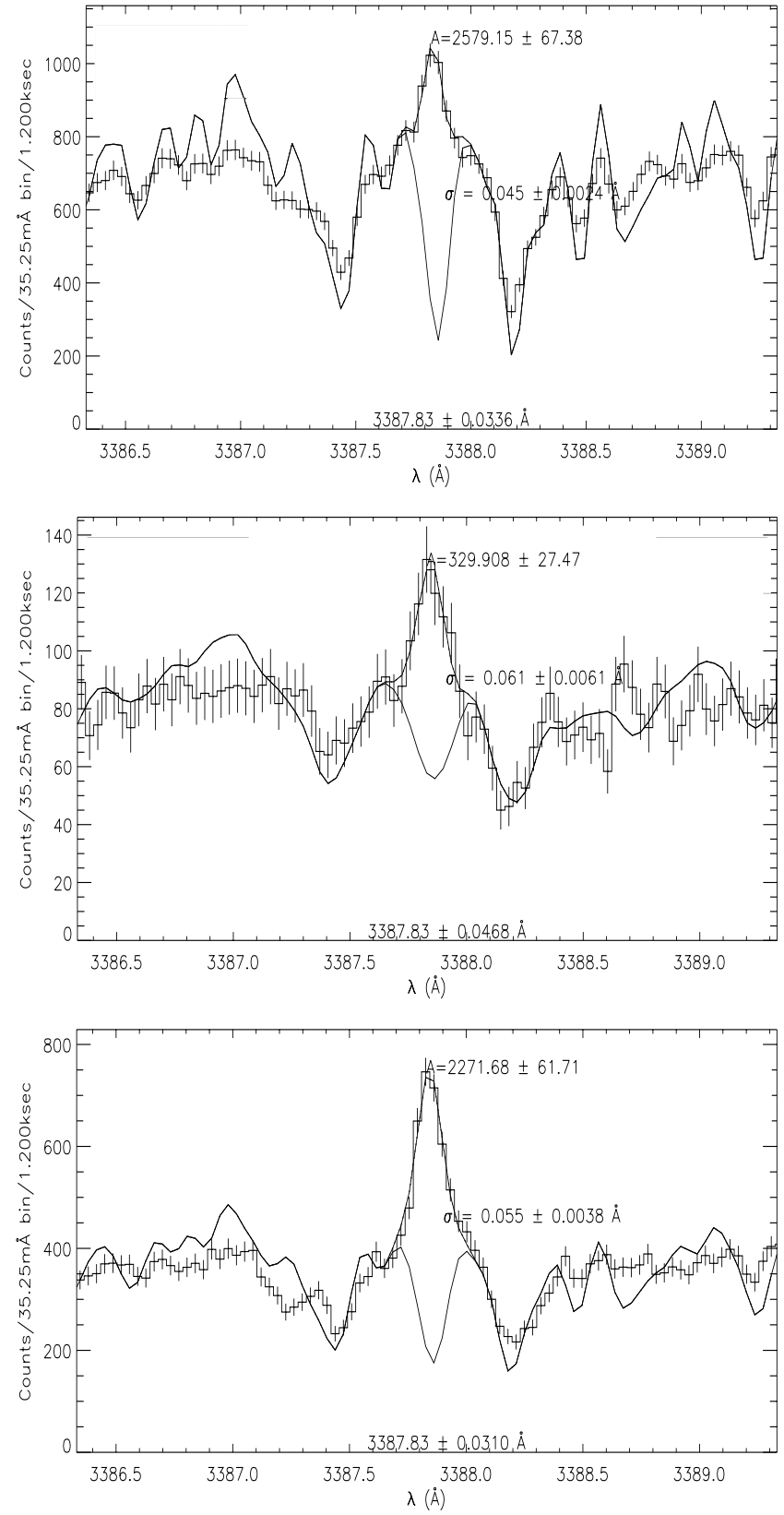

Fig. 6. The best fit of the $3387 \AA$ line for the stars AD Leo, AT Mic and YZ CMi with the rotational broadened spectra of GJ 229 as background (black line).

We used the CORA line fitting program to measure the Ti II lines and the Fe XIII line fluxes as described in Sect. 5.2; a listing of our fit results can be found in Table 5. The Fe XIII line was detected in all spectra. However, for the spectrum taken on March, 16th during the flare the fit results are ambiguous. One can obtain a good fit with two narrow lines or a narrow and a broad line, where the broad line has nearly the same central wavelength like the narrow line (i.e. the Fe XIII line is blueshifted). We assume that the fit with the lineshift of the Fe XIII line is the correct one, since there is definitely a second line and a physical interpretation with two narrow lines one of which is ascribed to Fe XIII with a halfwidth $\sigma$ of only $0.1 \AA$ is not meaningful due to temperature broadening. Therefore a fit 
Table 5. List of the line fluxes, central wavelength and half widths $\sigma$ measured with CORA. Due to the signal to noise ratio the computation of the error of the half width could not be done for every line.

\begin{tabular}{|c|c|c|c|c|c|}
\hline spectral block & Ti II (3372.80 ̊) & Ti II (3380.28 ̊̊) & Ti II (3383.77 ̊̊) & Ti II (3387.84 Å) & Fe XIII (3388.1 $)$ \\
\hline \multirow[t]{3}{*}{$2002-03-13$ 1st } & $137.7 \pm 16.3$ & $31.5 \pm 9.6$ & $83.2 \pm 12.8$ & $33.1 \pm 10.4$ & $115.6 \pm 26.3$ \\
\hline & $3372.77 \pm 0.05$ & $3380.27 \pm 0.04$ & $3383.74 \pm 0.04$ & $3387.82 \pm 0.02$ & $3387.88 \pm 0.17$ \\
\hline & $0.06 \pm 0.01$ & $0.03 \pm 0.01$ & $0.04 \pm 0.01$ & $0.02 \pm 0.01$ & 0.20 \\
\hline \multirow[t]{3}{*}{ 2002-03-13 2nd } & $290.1 \pm 21.6$ & $132.2 \pm 16.0$ & $215.4 \pm 18.8$ & $197.8 \pm 21.6$ & $201.0 \pm 33.0$ \\
\hline & $3372.78 \pm 0.04$ & $3380.25 \pm 0.04$ & $3383.73 \pm 0.03$ & $3387.83 \pm 0.03$ & $3388.02 \pm 0.09$ \\
\hline & $0.06 \pm 0.01$ & $0.05 \pm 0.01$ & $0.04 \pm 0.01$ & $0.05 \pm 0.01$ & 0.21 \\
\hline \multirow[t]{3}{*}{ 2002-03-14 } & $161.5 \pm 15.9$ & $61.4 \pm 12.6$ & $122.9 \pm 14.7$ & $81.6 \pm 16.2$ & $148.8 \pm 26.0$ \\
\hline & $3372.78 \pm 0.03$ & $3380.27 \pm 0.05$ & $3383.73 \pm 0.04$ & $3387.84 \pm 0.05$ & $3388.09 \pm 0.26$ \\
\hline & $0.04 \pm 0.01$ & $0.05 \pm 0.01$ & $0.04 \pm 0.01$ & 0.05 & 0.30 \\
\hline \multirow[t]{3}{*}{ 2002-03-15 1st } & $576.4 \pm 29.1$ & $295.7 \pm 23.4$ & $490.6 \pm 27.1$ & $302.2 \pm 28.1$ & $535.1 \pm 51.2$ \\
\hline & $3372.79 \pm 0.03$ & $3380.27 \pm 0.03$ & $3383.75 \pm 0.03$ & $3387.83 \pm 0.03$ & $3388.02 \pm 0.09$ \\
\hline & $0.04 \pm 0.01$ & $0.04 \pm 0.01$ & $0.04 \pm 0.01$ & $0.04 \pm 0.01$ & $0.26 \pm 0.02$ \\
\hline \multirow[t]{3}{*}{ 2002-03-15 2nd } & $437.7 \pm 24.8$ & $179.6 \pm 18.1$ & $315.0 \pm 21.8$ & $164.5 \pm 20.1$ & $265.7 \pm 39.0$ \\
\hline & $3372.80 \pm 0.03$ & $3380.27 \pm 0.03$ & $3383.76 \pm 0.03$ & $3387.82 \pm 0.04$ & $3388.19 \pm 0.17$ \\
\hline & $0.04 \pm 0.01$ & $0.04 \pm 0.01$ & $0.04 \pm 0.01$ & $0.05 \pm 0.01$ & 0.30 \\
\hline \multirow[t]{3}{*}{ 2002-03-16 1st } & $205.6 \pm 19.3$ & $68.1 \pm 12.5$ & $122.8 \pm 14.8$ & $57.2 \pm 14.3$ & $307.6 \pm 34.5$ \\
\hline & $3372.80 \pm 0.05$ & $3380.26 \pm 0.03$ & $3383.75 \pm 0.03$ & $3387.85 \pm 0.03$ & $3387.98 \pm 0.17$ \\
\hline & $0.06 \pm 0.01$ & $0.03 \pm 0.01$ & $0.04 \pm 0.01$ & $0.03 \pm 0.01$ & $0.24 \pm 0.02$ \\
\hline \multirow[t]{3}{*}{ 2002-03-16 2nd } & $125.5 \pm 15.3$ & $25.6 \pm 8.9$ & $65.5 \pm 10.8$ & $26.6 \pm 11.0$ & $134.8 \pm 26.6$ \\
\hline & $3372.82 \pm 0.05$ & $3380.28 \pm 0.04$ & $3383.76 \pm 0.03$ & $3387.84 \pm 0.03$ & $3387.88 \pm 0.14$ \\
\hline & $0.06 \pm 0.01$ & $0.03 \pm 0.01$ & $0.02 \pm 0.01$ & $0.02 \pm 0.01$ & 0.20 \\
\hline
\end{tabular}

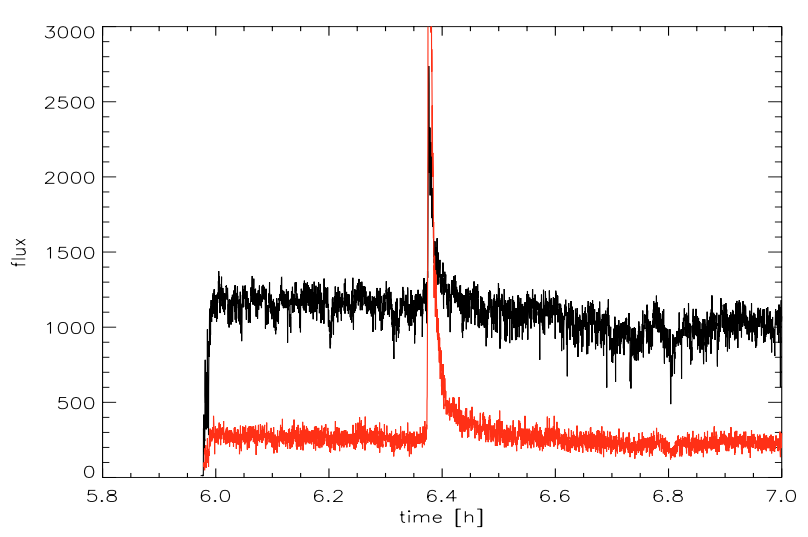

Fig. 7. Light curve of the flux of CN Leo on March, 16th, during the second observation block but otherwise like in Fig. 2. Clearly a major flare can be seen in both the red and the blue arm of the photometer.

with two narrow lines implies that there is no Fe XIII present. This can be possible, when the Fe XIII is ionized to higher ionisation stages during the flare. This is quite unlikely though, since we find no Fe XIV emission at $5303 \AA$ in CN Leo (see Sect. 8) even during the flare. Moreover the second emission line has to be explained otherwise. We favour therefore an interpretation with a narrow Ti II line and a broad blue shifted Fe XIII line. Line shifts are known to occur during flares, when the emitting material is raised in the atmosphere. The velocity towards the observer in the line of sight is about $20 \mathrm{~km} \mathrm{~s}^{-1}$ in this case. A blueshifted Fe XIII line during the flare lines in with the blueshift of the Fe XIII line we observed in LHS 2076 during the flare.

Although the photometer data for the spectra outside the major flare are rather constant, the corona must have undergone some variability as can be seen from Fig. 8, where the averaged spectra of each time series are shown. The Fe XIII line is most clearly seen in the spectrum of the first series taken on March, 15th, where the continuum is relatively high and the Ti II line is very intense, although in the photometer data no notable flux variability is seen besides the usual flickering. In the other spectra the line does not show that clearly, in fact the line would be undetectable if it were only a bit weaker or if one chose a higher background level. Thus even for CN Leo the Fe XIII line need not to be a persistent feature, and the high variability during quiescence is suggestive of the microflaring as proposed for the heating of stellar coronae (Kashyap et al. 2002). Also the chromospheric Ti II line shows major variability even on a timescale of hours. For example, on March 13th the amplitude of the Ti II lines increased by a factor of more than two over a time span of only two hours. Therefore also the chromosphere must have undergone rapid and large changes.

\section{X-ray and Fe XIII line fluxes}

Using the standard star HD 49798 for flux calibration, we measured absolute fluxes in the Fe XIII 3388.1 line for the stars CN Leo, GL Vir and for LHS 2076 (during the flare). Since the standard star was observed only once per night, we estimate an error in this absolute flux calibration up to a factor 

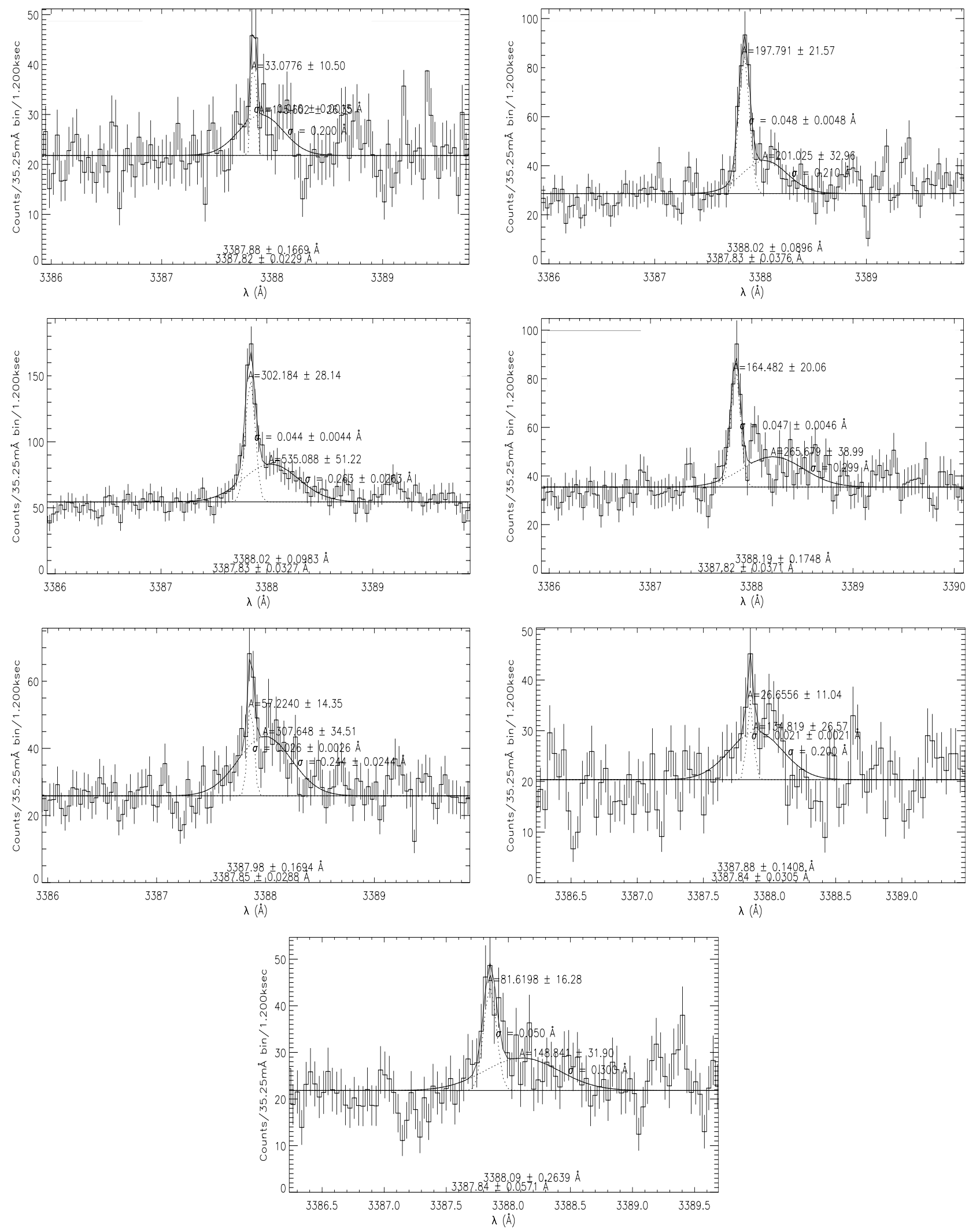

Fig. 8. Best fit of the Ti II line together with the Fe XIII line in the averaged spectral series. At the top the two spectra taken on March, 13th (left: start time 2.3 UT right: start time 4.2 UT), beneath the two spectra taken on March, 15th (left: start time 3.7 UT, right: start time 6.4 UT), beneath the spectra taken on March, 16th (left: start time 3.9 UT, right: start time 5.9 UT). The single spectrum at the bottom was taken on March, 14th (start time 3.1 UT). The horizontal line in each spectrum indicates the background used for the line fitting. 


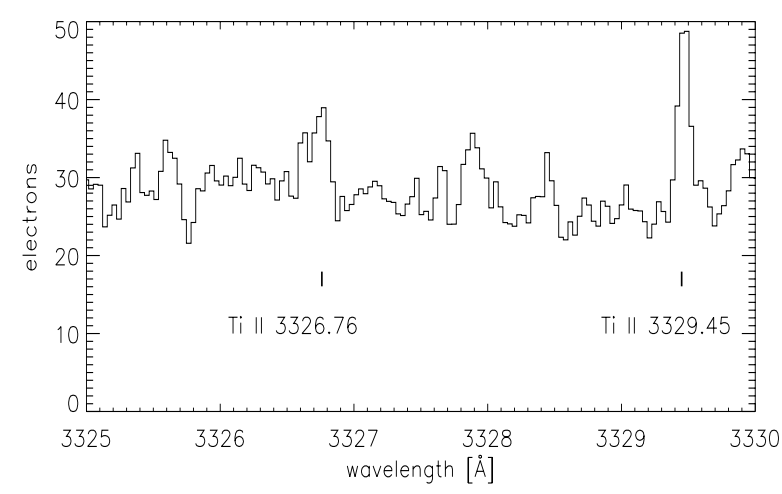

Fig. 9. The spectrum of CN Leo around the Ca XII line. Two Ti II lines are identified; the other faint emission features are unidentified.

of two. Broad band X-ray fluxes for these stars were obtained from broad band count rates and should have (systematic) errors of about $50 \%$, resulting from the adopted count rate to energy flux conversion. Also note that for none of our stars do we have simultaneous optical and X-ray observations. The measured average fluxes for the one hour duration spectra of CN Leo range from $3.2 \times 10^{-15} \mathrm{~cm}^{-2} \mathrm{~s}^{-1}$ in the first spectrum taken on $2002-03-13$ to $1.4 \times 10^{-14} \mathrm{erg} \mathrm{cm}^{-2} \mathrm{~s}^{-1}$ in the first spectrum on 2002-03-15. For the Fe XIII line flux of GL Vir we computed $3.9 \times 10^{-15} \mathrm{erg} \mathrm{cm}^{-2} \mathrm{~s}^{-1}$, and for the flare spectrum of LHS 2076 the measured flux is $9.8 \times 10^{-15} \mathrm{erg} \mathrm{cm}^{-2} \mathrm{~s}^{-1}$. Using distances of $2.4 \mathrm{pc}$ for CN Leo $6.5 \mathrm{pc}$ for GL Vir and $5.2 \mathrm{pc}$ for LHS 2076 (Oppenheimer et al. 2001) these fluxes result in line luminosities of $2.0 \times 10^{24}$ up to $9.6 \times 10^{24} \mathrm{erg} \mathrm{s}^{-1}$ for CN Leo, $1.9 \times 10^{25} \mathrm{erg} \mathrm{s}^{-1}$ for GL Vir and $3.2 \times 10^{25} \mathrm{erg} \mathrm{s}^{-1}$ for LHS 2076. Comparing these numbers with the non-simultaneously measured X-ray luminosities one then finds a ratio of broad band $\mathrm{X}$-ray to line luminosity in the range 3012 to 628 for CN Leo, 235 for GL Vir and of 124 for LHS 2076.

If one takes CN Leo as a prototype showing Fe XIII emission the ratios of GL Vir and LHS 2076 are too low. However, for LHS 2076 the Fe XIII was measured during a flare, while the X-ray luminosity was measured during quiescence and is therefore presumably too low leading to an incorrect (lower) ratio. In the case of GL Vir the line was measured during quiescence and the low ratio adds therefore to the interpretation, that the line is actually the Ti II line broadened by the higher rotation rate we found for GL Vir.

\section{Search for other forbidden coronal lines in CN Leo}

After the detection of the Fe XIII line in CN Leo the question arises if there is evidence for other forbidden coronal lines. A catalog of such lines can be found e.g. in Allen (1973). Out of this we investigated the following two lines more closely: Ca XII at $3327 \AA$ and Fe XIV at $5303 \AA$.

Using the CHIANTI software package (Young et al. 2003) we computed emissivity ratios for the lines mentioned above to the Fe XIII line, which are proportional to the flux ratios of the lines. All computations were done assuming solar photospheric abundances since we do not know anything specific about the coronal abundances of very low mass stars. The

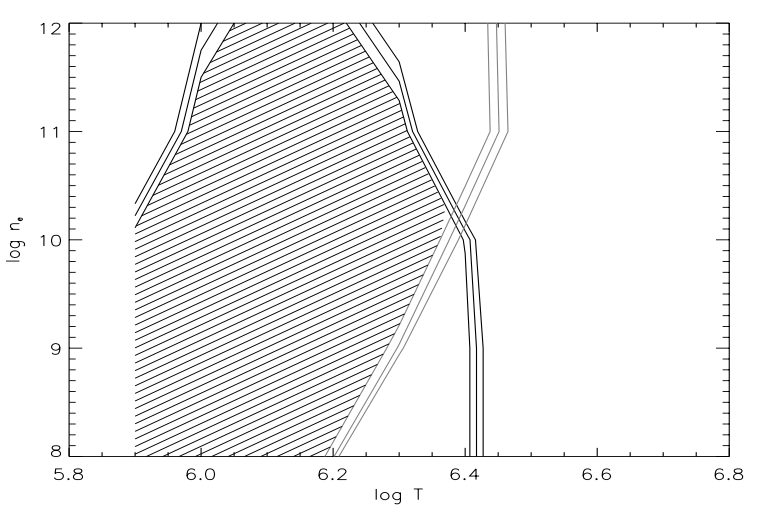

Fig. 10. Contour plot of the flux ratios between the CaXII and the Fe XIII line (black) for a set of temperatures and electron densities and for the ratios of the Fe XIV and the Fe XIII line (grey). The inner line represents a ratio of 0.4 , the middle line a ratio of 0.5 and the outer line a ratio of 0.6 for Ca XII and for Fe XIV the inner line represents a ratio of 5.0, the middle line a ratio of 5.5 and the outer line a ratio of 6.0. The shaded area denotes the parameter set that is in agreement with the non-detection of Ca XII and Fe XIV.

ionisation balance was computed using the ionisation ratios found by Mazzotta et al. (1998). The computed flux ratios proved to be quite sensitive on the ionisation balance whose choice can influence the computed flux ratios by more than a factor of two while the chosen abundances have less influence.

\subsection{Analysis of the Ca XII line}

A plot of the region around the Ca XII line is shown in Fig. 9. Besides the two Ti II lines the other faint emission features are unidentified, but none of them is likely to be caused by Ca XII because the line width is too narrow for a line formed at temperatures of about $\log T \sim 6$ for which one would expect a halfwidth of $0.2 \AA$. While a line consisting of 50 counts and a halfwidth of $0.2 \AA$ can easily be hidden in the spectrum a line with 100 counts should be possible to detect. This leads to restrictions for the possible temperatures and densities. Since we measured 200 counts in the Fe XIII line at $3388 \AA$ for $\mathrm{CN}$ Leo averaging all our spectra, the highest possible ratio between the two lines that is in agreement with a non-detection is about 0.5 , which excludes temperatures higher than $\log T=6.4$ and lower than $\log T=6.0$. A more detailed discussion is given in the next subsection together with the restrictions drawn from Fe XIV.

\subsection{Analysis of the Fe XIV line}

We carefully searched for the Fe XIV line at $5303 \AA$. Since the line could be hidden in the molecular lines dominating this wavelength region we compared the CN Leo spectra to each other and to the LHS 2034 averaged spectrum, assuming that this star does not display the Fe XIV line. But no significant difference could be found. Therefore we conclude, that no Fe XIV emission can be seen in CN Leo.

Out of the absence of the Fe XIV and the CaXII emission lines the possible temperatures and densities can be estimated. To be in agreement with a non-detection for the Ca XII line the 
flux ratio must be lower than about 0.5 and for the Fe XIII lower than about 5.5. In Fig. 10 a contour plot of the ratio between the CaXII and the Fe XIII and the Fe XIV and the Fe XIII line is given. From this plot one can exclude temperature lower than $\log T=6.0$ and higher than $\log T=6.2$ for low densities, $\log T=6.3$ for higher densities and $\log T=6.4$ for very high densities. Therefore the line formation of the Fe XIII line takes place around the peak formation temperature of $\log T=6.2-$ what would be expected from the beginning. For the densities the plot is not very restrictive. Even densities as high as $N_{\mathrm{e}}=$ $10^{12} \mathrm{~cm}^{-3}$ can be possible if the temperature is close enough to the peak formation temperature.

\section{Discussion and conclusions}

Our study reveals high and rapid variability of the Fe XIII line on the two stars CN Leo and LHS 2076. While the former seems to have coronal temperatures high enough even in its quiescent state to show the Fe XIII line, the latter does not.

In addition to these two absolutely secure detections GL Vir and UV Cet may exhibit the Fe XIII line, too. For Prox Cen the situation is unclear. In the stars AD Leo, AT Mic, YZCMi and FN Vir no Fe XIII line could be found. In the stars DX Cnc, LHS 292 and the quiescent LHS 2076 no Fe XIII line could be found as well. That may be due to the low signal to noise ratio but simulation strongly suggest that these stars are not active enough to show the Fe XIII line. Rather surprisingly no Fe XIII line could be found in the flaring LHS 2034, either. So there are at most four detections of Fe XIII in a sample of 16 or 13 stars, respectively if one does not count the two double stars LHS 428 and LHS 6158 and the template GJ 229A. This might seem not very much at first glance since all are very active stars. However, one of the the goals of the project was to observe a wide range of spectral types to determine for which stars a detection of Fe XIII is feasible at all. And if the case of LHS 2076 applies to the other stars as well and the corona is only hot enough to show the Fe XIII line during major flares we would only expect one more detection, since LHS 2034 showed a flare, too. But in contrast to the two flares on CN Leo and LHS 2076 this was a long duration flare, whose decays lasted longer than half an hour and we would expect different behavior of the spectral lines in the course of such a different type of flare.

Moreover the Fe XIII line may have been detected for some of the stars with a better signal to noise ratio. This can be estimated for AD Leo where from EUVE observations a flux at Earth of $2.69 \times 10^{-4}$ photons $\mathrm{cm}^{-2} \mathrm{~s}^{-1}$ has been measured for the 203.83 A line of Fe XIII (Sanz-Forcada \& Micela 2002). Using atomic data from the CHIANTI database (Young et al. 2003), we compute a flux ratio of the two lines of $\frac{F_{3388}}{F_{203}}=0.02 \ldots 0.05$ assuming coronal densities in the range of $10^{8}$ up to $10^{10} \mathrm{~cm}^{-3}$ and temperatures in the range of 1 up to $2 \times 10^{6} \mathrm{~K}$. This translates into a line flux of $5.2 \times 10^{-16} \cdots 1.3 \times 10^{-15} \mathrm{erg} \mathrm{cm}^{-2} \mathrm{~s}^{-1}$ in the Fe XIII line at $3388 \AA$, which in turn would correspond to an amplitude of at most 26 counts in this line (for a $0.3 \mathrm{~h}$ integration, which is the typical exposure time for all of our spectra); clearly, such flux levels can easily be hidden in the spectrum. If the Fe XIII line at $203.83 \AA$ is blended with other iron lines the expected amplitude would be even less. Therefore data with much better signal to noise are required to settle this question.

$\mathrm{CN}$ Leo is unique in the sample when it comes to clear exhibition of the Fe XIII line in quiescent state, which we ascribe to a high level of microflaring, since the coronal line is quite variable. This variability of the corona while the photospheric flux measured with the photometer is quite constant calls for a parallel observation in X-rays with Chandra or XMM-Newton, since the coronal variability should lead to X-ray variability. This high level of variability in the basic coronal emission is surprising, but it finds it counterpart in the high level of chromospheric emission in the hydrogen emission lines of the Balmer series which are clearly seen up to $\mathrm{H}_{24}$. This is in contrast to the Sun where even in flares the Balmer lines are only excited up to $\mathrm{H}_{16}$ (Švestka 1972).

Though the variability of Fe lines for the Sun was studied extensively in X-ray wavelengths by the satellites SOHO and TRACE the variability of the emission in Fe XIII lines was not studied to our knowledge, yet. However for the forbidden optical green Fe XIV line at $5303 \AA$ there are measurements by the SOHO instrument LASCO (Large Angle Spectrometric Coronagraph) (Wood et al. 1998). Observing quiescent coronal limb structures Wood et al. found statistically significant quasi-steady brightening on timescales of at least an hour. This is about the same timescale on which we observed the fading of the Fe XIII line in CN Leo on March, 15th from a very pronounced to a barely detectable line profile, which took less than three hours.

Acknowledgements. We thank Dr. Jan-Uwe Ness supplying to us his CORA line fitting program and providing technical support.

\section{References}

Allen, C. W. 1973, University of London (The Athlone Press) Audard, M., Güdel, M., \& Skinner, S. L. 2003, ApJ, 589, 983

Berger, E. 2002, ApJ, 572, 503

Delfosse, X., Forveille, T., Perrier, C., \& Mayor, M. 1998, A\&A, 331, 581

Flower, D. R., \& Pineau des Forets, G. 1973, A\&A, 24, 181

Güdel, M. 2002, ARA\&A, 40, 217

Hünsch, M., Schmitt, J. H. M. M., Sterzik, M. F., \& Voges, W. 1999, A\&AS, 135, 319

Kashyap, V. L., Drake, J. J., Güdel, M., \& Audard, M. 2002, ApJ, 580, 1118

Mazzotta, P., Mazzitelli, G., Colafrancesco, S., \& Vittorio, N. 1998, A\&AS, 133, 403

Mohanty, S., \& Basri, G. 2003, ApJ, 583, 451

Ness, J.-U., \& Wichmann, R. 2002, AN, 323, 129

Oppenheimer, B. R., Golimowski, D. A., Kulkarni, S. R., et al. 2001, AJ, 121, 2189

Pettersen, B. R. 1985, A\&A, 148, 151

Sanz-Forcada, J., \& Micela, G. 2002, A\&A, 394, 653

Schmitt, J. H. M. M. 1997, A\&A, 318, 215

Schmitt, J. H. M. M., Fleming, T. A., \& Giampapa, M. S. 1995, ApJ, 450,392

Schmitt, J. H. M. M., \& Liefke, C. 2002, A\&A, 382, L9

Schmitt, J. H. M. M., \& Wichmann, R. 2001, Nature, 412, 508

Stepanov, A. V., Fuerst, E., Krueger, A., et al. 1995, A\&A, 299,739

Švestka, Z. 1972, ARA\&A, 10, 1

Wood, B. E., Karovska, M., Cook, J. W., et al. 1998, ApJ, 505,432

Young, P. R., Del Zanna, G., Landi, E., et al. 2003, ApJS, 144, 135 